\title{
OSTEOPENIA IN CHILDREN WITH MALABSORPTION SYNDROM
}

\author{
Tania Elena Rusu' ${ }^{1}$ Evelina Moraru², Laura Bozomitu', \\ Dana Teodora Anton Paduraru', Lucretia Anghel ${ }^{3}$, Aurica Rugina ${ }^{1}$ \\ ${ }^{1}$ Department of Pediatrics, "Gr. T. Popa" University of Medicine and Pharmacy, Iasi \\ ${ }^{2} I I$ Pediatric Clinic, "Sf. Maria" Emergency Children's Hospital, Iasi \\ ${ }^{3}$ Department of Internal Medicine, "Dunarea de Jos" University of Medicine, Galati
}

\begin{abstract}
Introduction. Malabsorption syndromes result in the disturbance of bone normal development and function. Objective. The assessment of bone density in children with malabsorption syndromes. The analysis of risk factors for osteopenia. The correlation between osteopenia, nutritional markers and bone metabolism markers. Material and method. 118 children with malabsorbtion syndromes of different etiologies, mainly Celiac disease (41 cases) and Cystic fibrosis (14 cases). Bone density was assessed by Quantitative Ultrasonography (QUS) with a Sunlight Omnisense Ultrasonometer 7000P. QUS was performed at two sites - radius (86 cases) and tibia (78 cases). 25hydroxivitamin D was measured in 10 cases by RIA method. Statistical analysis was made using SPSS for Windows.

Results. Osteopenia was present in $32 \%$ cases. Decreased bone density at the radius was associated with the celiac syndrome. Osteopenia at the tibia was associated with cystic fibrosis. Osteopenia was more frequent in girls. Osteopenia was related to the duration of the disease. Osteopenia wasn't related to BMI. Radius Z-score positively correlated to alkaline phosphatase levels and tibia Z-score to serum cholesterol levels. Radius and tibia Z-score negatively correlated with inflammatory marker levels. In children with celiac disease, the value of anti-transglutaminase antibodies was negatively correlated to radius and tibia Z-score values. $25(\mathrm{OH})$ vitamin D values were deficient in 8 patient and insufficient in 2, but its values didn't correlate to radius/tibia Z-score. Clinical, biological and radiological signs of rickets were found in $35 \%$ of patients with osteopenia.

Conclusions. Osteopenia was found in $1 / 3$ of patients with malabsorption syndromes of the studied group. In $30 \%$ of patients, clinical, biological and radiologic rickets signs were present. $25(\mathrm{OH})$ vitamin D values didn't correlate with the Z-score. A negative correlation between bone parameters and inflammation markers and anti-transglutaminase antibodies values was observed.
\end{abstract}

Keywords: osteopenia, malabsorption syndromes, celiac disease

\section{INTRODUCTION}

Osteopenia («greek osteon» oston = bone, penia $=$ lack, poorness) defines a low bone mass with histological bone tissue deficit and radiologic signs of bone mass reduction.

Bone density evaluation is mandatory in children, because peak bone mass and the lifetime bone capital is achieved mainly in childhood.

The main category of diseases that can affect bone mass in childhood are: endocrine diseases (growth hormone deficit, sex hormones deficit, glucocorticoid excess, thyroid hormone excess), rheumatologic diseases (juvenile idiopathic arthritis, systemic lupus erythematosus), chronic hepatic diseases, chronic renal diseases, chronic inflamma- tory bowel disease, malabsorption syndromes, haematological diseases (thalassemia, sickle cell disease, haemophilia, malignant hemopathies, a variety of genetic disorders (damages of collagen synthesis genes, alteration of vitamin D receptor, storage diseases, homocystinuria, porphyria).

Clinical entities having as clinical features chronic diarrhoea, abdominal distension and growth failure, grouped as Malabsorption syndromes include, among many homeostatic imbalances, the disturbance of bone normal development and function $(1,2)$. Bone density decrease, associated with the clinical-biological features of rickets may be early and they are markers of the malabsorption syndrome severity. The main causes of low bone mass in malabsorption syndromes are hypocal-

Corresponding author:

Tania Elena Rusu, “Sf. Maria” Emergency Children's Hospital, 62 Vasile Lupu Street, lasi

E-mail: taniaelenarusu@gmail.com 
caemia (and other mineral deficiencies), hypovitaminosis $\mathrm{D}$, deficit of nutrients for the synthesis of collagen matrix, chronic inflammation via inflammatory cytokines, unbalance between factors that regulate the Bone Multicellular Unit-BMU depending on the mechanism, duration and severity of the clinical entity resulting in malabsorption syndrome. For example, in celiac disease, bone mass reduction is due to calcium, vitamin D and protein malabsorption secondary to enterocyte destruction, proinflammatory cytokine release and their unbalancing effect on the BMU synthesis/resorbtion cycle, endocrine dysfunction (secondary hyperparathyroidism, androgens/estrogens unbalance), nutritional deficiencies related to the gluten-free diet, the direct effect of the anti-transglutaminase antibody on bone, autoimmune disturbances (diabetes, autoimmune thyroiditis), genetic factors (genetic polymorphism of Il-1b gene), treatment with corticosteroids.

\section{OBJECTIVES}

- Assessment of osteopenia incidence in children with malabsorption syndromes.

- Analysis of risk factors for osteopenia.

- The correlation between osteopenia, nutritional markers and bone metabolism markers.

\section{MATERIAL AND METHOD}

The prospective study included 118 children diagnosed in the Pediatric Clinics of "Sf. Maria" Children's Emergency Hospital Iasi, with malabsorption syndromes of various etiologies. Most cases were represented by celiac disease (41 cases) followed, in order of the frequency of malabsorption, of unspecified etiology (16 cases), cystic fibrosis (14 cases), intolerance to cow's milk protein (11 cases), eosinophilic gastroduodenitis (7 cases), hypogammaglobulinemia (6 cases), selective Immunoglobulin A deficiency (5 cases), lambliasis (5 cases), celiac syndrome (3 cases), Ehlers Danlos syndrome ( 2 cases), Immunoglobulin $\mathrm{G}$ deficiency (1 case) and intestinal resection (1 case).

Assessment of bone density was performed with Quantitative Ultrasonography using a Sunlight Omnisense 7000P Osteodensitometer. The measured parameters were the speed of sound through bone (SOS) and the Z-score. A Z-score below-1 was considered osteopenia. The measurements were carried out at the radius in 86 cases and at the tibia in 78 cases. 25hydroxivitamin D level assessment was possible in 10 cases using RIA. Vitamin D deficiency is defined as a serum value under $20 \mathrm{ng} / \mathrm{ml}$. Values between 21 and $29 \mathrm{ng} / \mathrm{ml}$ are considered vitamin D insufficiency.

Statistical analysis was performed with the SPSS program for Windows.

Correlations of the Z-score were made with malabsorption syndrome etiology, demographic parameters: age, sex, duration of disease, nutritional indices: BMI, nutritional biological parameters: total blood serum protein, albumin, hemoglobin, serum total lipids, blood cholesterol, magnesemia, blood iron, blood sugar; the parameters of calciumphosphor metabolism: total calcium, ionic calcium, total phosphorus, alkaline phosphatase; inflammatory markers: fibrinogen, ESR, anti-transglutaminase antibody value. Correlations were made through the Chi-square test, Mann-Whitney test, $t$ test and Anova test, regression lines and Pearson coefficient.

\section{RESULTS}

Osteopenia was present in $32.2 \%$ of cases, respectively $38 / 118$ cases with malabsorption syndrome of various etiologies.

In celiac disease (the most numerous subgroup), osteopenia had a frequence of $35,89 \%$, followed by cystic fibrosis $(50 \%)$, malabsorption of unknowkn etiology, eosinophilic gastroduodenitis, Ehlers Danlos syndrome and hypogammaglobulinemia. In children with post-gastroenteritis syndrome, osteopenia was absent (Fig. 1). Evaluation of the relationship between the decrease of the Z-score under -1 at the radius and the various diagnoses reveals that the only diagnosis that was associated with decreased bone density at the radius was the celiac syndrome (Chi-square test, $\mathrm{p}=0.047$ ). Z-score at the tibia below-1 was associated with the diagnosis of cystic fibrosis at the Chi-square test $(p=0.047)$.

No statistically significant differences were recorded between radius Z- score values in different diagnoses. Significantly higher Z-score values at the tibia were obtained in post-gastroenteritis syndrome. Significantly lower values of tibia Z-score were obtained in patients with cystic fibrosis (Table 1).

Osteopenia was more frequent in preschool and school age. The average age of cases with osteopenia was higher than that of patients with normal bone mass (Fig. 2), statistically significant difference at the Mann-Whitney test $(\mathrm{p}=0.015)$. 


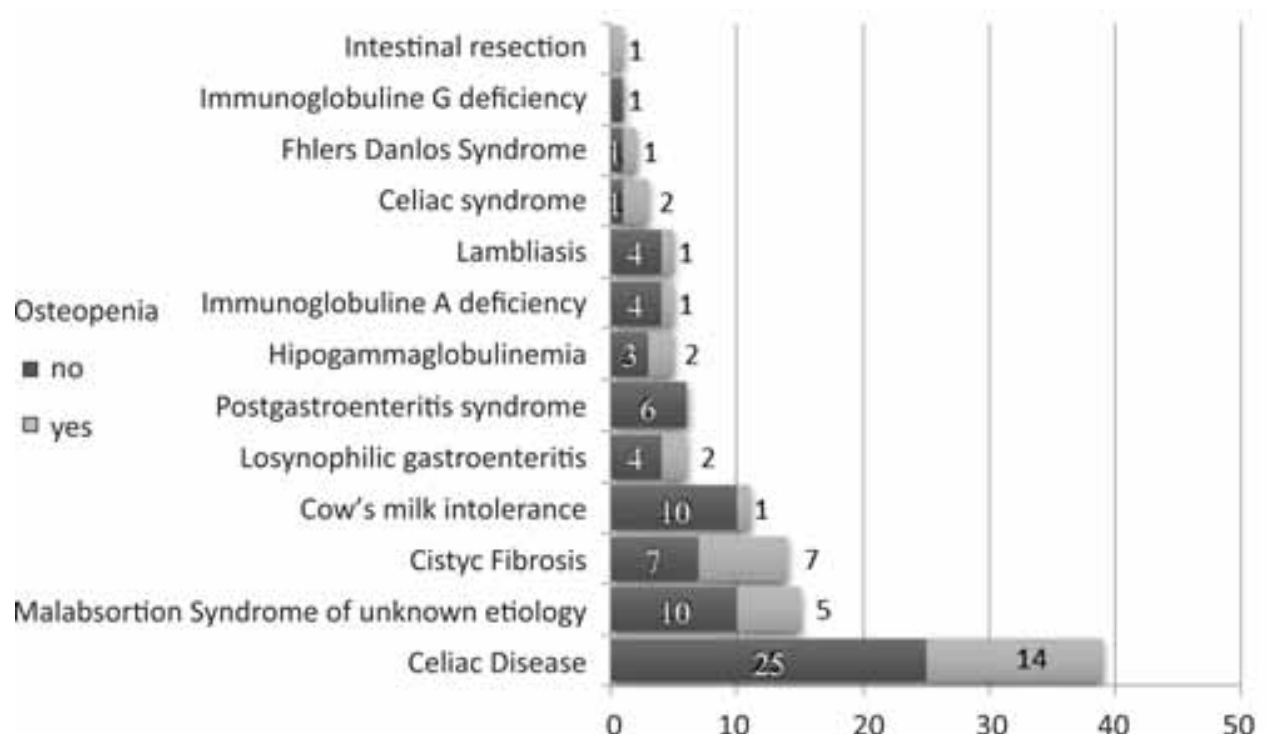

FIGURE 1. The presence of osteopenia vs. malabsorption syndrome etiology

TABLE 1. Radius and tibia Z-score t test comparison of different diagnosis

\begin{tabular}{|l|c|c|}
\hline DIAGNOSIS & T-test for radius Z-score & T-test for tibia Z-score \\
\hline Celiac disease & $\mathrm{p}=0.220$ & $\mathrm{p}=0600$ \\
\hline Malabsorption syndrome of unknown etiology & $\mathrm{p}=0.980$ & $\mathrm{p}=0.622$ \\
\hline Cystic Fibrosis & $\mathrm{p}=0.154$ & $\mathrm{p}=0.024$ \\
\hline Cow's milk intolerance & $\mathrm{p}=0.439$ & $\mathrm{p}=0.683$ \\
\hline Eosinophilic gastroenteritis & $\mathrm{p}=0.325$ & $\mathrm{p}=0.711$ \\
\hline Post-gastroenteritis syndrome & $\mathrm{p}=0.067$ & $\mathrm{p}=0.012$ \\
\hline Hipogammaglobulinemia & $\mathrm{p}=0.740$ & $\mathrm{p}=0.873$ \\
\hline IgA deficiency & $\mathrm{p}=0.653$ & $\mathrm{p}=0.561$ \\
\hline Lambliasis & $\mathrm{p}=0.613$ & $\mathrm{p}=0.052$ \\
\hline Celiac syndrome & $\mathrm{p}=0.264$ & $\mathrm{p}=0.639$ \\
\hline Ehlers Danlos Syndrome & $\mathrm{p}=0.477$ & $\mathrm{p}=0.689$ \\
\hline IgG deficiency & - & $\mathrm{p}=0.060$ \\
\hline Intestinal resection & 0433 & \\
\hline
\end{tabular}

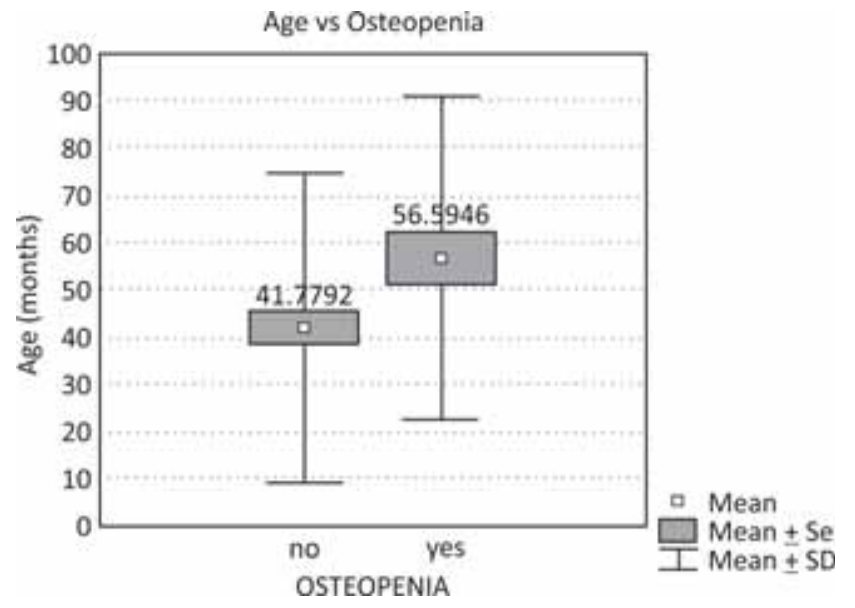

FIGURE 2. The average value of age (months) in cases with and without osteopenia

Average duration of the diseases accompanied by osteopenia was 10,61 months, compared to 7,13 months in those without osteopenia $(\mathrm{p}=0.143)$.
Osteopenia was more frequent in girls, 36\%, than in boys, $28 \%(p=0.377)$.

No statistically significant association was observed between poor nutrition, frequent in malabsorption syndromes as a result of the complex digestive affliction and impairment of supply, and osteopenia, in this uneven study $(\mathrm{p}=0.065)$. Average BMI values in patients with osteopenia were 15,21 compared to 14,26 in those without osteopenia.

The relationship between radius/tibia Z-score and some biological markers of the malabsorption syndrome using Pearson correlation coefficient, Anova test and regression lines, showed the following correlations:

- Positive between radius Z-score and alkaline phosphatase.

- Negative between radius Z-score and fibrinogenemia. 
- Negative between tibia and fibrinogenemia.

- Negative between tibia Z-score and ESR values.

- Positive between tibia Z-score and serum cholesterol values.

Globaly, osteopenia was associated with clinical signs of rickets in $30 \%$ of cases, biological markers of rickets in $34.7 \%$ and radiological changes in $35.7 \%$.

The metabolite 25hydroxivitamin D was dosed in 10 patients and the results are presented in Table 2. Values were assigned as vitamin D insufficiency in 2 cases and vitamin $\mathrm{D}$ deficiency in 8 cases.

TABLE 2. $25 \mathrm{OH}$ vitamin $D$ values in patients with malabsorption syndromes

\begin{tabular}{|l|c|c|}
\hline DIAGNOSIS & 25 OH vitamin D & Z-SCORE (RADIUS) \\
\hline Cystic Fibrosis & $\begin{array}{c}\text { Below the limit of } \\
\text { detection }\end{array}$ & .32 \\
\hline Cystic Fibrosis & $\begin{array}{c}\text { Below the limit of } \\
\text { detection }\end{array}$ & -0.16 \\
\hline $\begin{array}{l}\text { Malabsorption } \\
\text { syndrome }\end{array}$ & 19.89 & 1 \\
\hline Celiac disease & 19.86 & 0.65 \\
\hline Celiac disease & 17.68 & 0.21 \\
\hline Cystic Fibrosis & 11.93 & -1.06 \\
\hline Celiac disease & 26.99 & 1.54 \\
\hline Cystic Fibrosis & Below the limit of \\
detection & -0.5 \\
\hline $\begin{array}{l}\text { Post-lambliasis celiac } \\
\text { syndrome }\end{array}$ & 24.16 & 1.15 \\
\hline Celiac disease & 18.41 & 3.84 \\
\hline
\end{tabular}

The evaluation of the correlation between antitransglutaminase antibodies value and the radius and tibia Z-score using Anova test and Pearson correlation coefficient showed a strong negative correlation between anti-transglutaminase antibody values and radius $\mathrm{Z}$-score and a mild negative correlation between anti-transglutaminase antibody values and tibia $Z$-score.

\section{DISCUSSION}

Bone density studies in patients with celiac disease have reported a decrease in bone mass in $75 \%$ of newly diagnosed patients, who are not on glutenfree diet $(3,4)$. In celiac disease, immune-mediated enterocyte destruction leads to a decrease in the absorption surface, having as consequence the impaired absorption of calcium, vitamin $\mathrm{D}$ and protein. This is reflected in reduced serum calcium values, which results in reactive hyperparathyroidism $(5,6)$. Hyperparathyroidism leads to increased osteoclastic resorbtion and 1 alpha hydroxilase en- zyme activity amplification, resulting in the augmentation of 1,25 dihydroxyvitamin $\mathrm{D}$ synthesis. The stimulation of the intestinal absorption of calcium by 1,25 dihydroxyvitamin $\mathrm{D}$ is ineffective because the immature enterocytes possess very low levels of calbindin, a calcium transporter (5).

Decreased serum levels of 25 hydroxyvitamin D may be due not only to the impaired absorption, but also to the decreased intake and low plasma halflife time due to the excessive transformation in 1,25 dihydroxyvitamin D by elevated serum PTH levels. Elevated serum levels of 1,25 dihydroxyvitamin D have a harmful effect on bone metabolism, promoting bone resorbtion $(5,6)$.

The presence of clinical, biological and radiological signs of rickets in about $35 \%$ of patients with osteopenia suggest that hypovitaminosis D and reactive hyperparathyroidism are implicated in the etiopathogenesis of osteopenia in about $35 \%$ of patients in the studied group. Zanchi et al (2008) report disturbed calcium metabolism in about $50 \%$ of 54 patients with celiac disease. Half of them had osteopenia in DEXA Osteodensitometry (7). The present study showed similar results, the association of two biological signs of calcium and phosphor metabolism disturbance was present in 50\% of the 41 patients with celiac disease and half of these had osteopenia in QUS Osteodensitometry.

Recently, research has focused on establishing the role of cytokines in the pathogenesis of bone damage from celiac disease. Alteration of the osteosynthesis/resorbtion ballance play an important role in the pathogenesis of osteopenia in celiac disease, because of the increased levels of osteoclast activatory cytokines (IL-1,IL-6, TNF $\alpha$ ), and low levels of osteoclast inhibitory cytokines (IL-18 şi IL-12) $(5,8)$.

The negative correlation between radius and tibia Z-score and fibrinogen and ESR values in our study advocates the role of inflammation in the etiopathogenesis of osteopenia in some of the patients with malabsorption syndrome included in the study. Bianchi et al (2008) point out the role of inflammation in celiac bone mass alteration pathogenesis (9). Taranta et al (2004) highlight the role of osteoclast activation by pro-inflammatory cytokines in celiac disease osteopenia (10).

Anti-transglutaminase IgA antibodies act against bone tissue and their level correlates with the level of bone mass reduction, suggesting that they play a role in the pathogenesis of osteopenia in celiac disease (11). The strong negative correlation between anti-transglutaminase antibody values and radius/ tibia Z-score values supports the hypothesis of the 
involvement of these antibodies in the alteration of bone mass in the studied group.

Osteopenia, a common cystic fibrosis complication, alters the quality of life of these patients, as their life expectancy grows. Many studies have documented the bone mass reduction in these patients, that correlated with pancreatic disease and pulmonary impairment severity, nutritional status, age, stage of puberty, presence and duration of corticoid therapy, physical activity (12-17). Osteopenia was found in half of the cystic fibrosis patients in the studied group, but the small sample did not permit statistical correlations.

\section{CONCLUSIONS}

Osteopenia was found in at least $1 / 3$ of patients with malabsorption syndromes of the studied group

\section{REFERENCES}

1. Siddiqui Y., Osazande A.S. Selected disorders of malabsorption. Prim Care. 2011 Sep. 38(3): 395-414.

2. Precup L. Sindromul de malabsorbtie. In: Lazăr D: Patologie pediatrica la copil, vol II, Editura Național, Bucureşti 1999:205-232.

3. Kavak U.S., Yuce A., Kocak N., Demir H., Saltik I.N., Gurakan F., Ozen $\mathrm{H}$. Bone mineral density in children with untreated and treated celiac disease. J Pediatr Gastroenterol Nutr 2003:37(4): 434-436.

4. Corazza G.R., Di Sario A., Cecchetti L., Tarozzi C., Corrao G., Bernardi M., Gasbarrini G. Bone mass and metabolism in patients with celiac disease. Gastroenterology 1995:109:122-128.

5. Krupa Kozak U. Pathologic bone alterations in celiac disease: Etiology, epidemiology and treatment. Nutr 2014: 30: 16-24.

6. Keaveny A.P., Freaney R., McKenna M.J., Masterson J., O'Donoghue D.P. Bone remodeling indices and secondary hyperparathyroidism in celiac disease. Am J Gastroenterol 1996:91:1226-1231.

7. Zanchi C., Di Leo G., Ronfani L., Martelossi S., Not T., Ventura A. Bone metabolism in celiac disease. J Pediatr. 2008: 153(2):262-5.

8. Garrote J.A., Gomez-Gonzalez E., Bernardo D., Arranz E., Chirdo F. Celiac disease pathogenesis: the proinflammatory cytokine network. J Pediatric Gastroenterol Nutr 2008;47(Suppl 1):27-32.

9. Bianchi M.L., Bardella M.T. Bone in celiac disease. Osteoporos Int 2008:19(12):1705-16.

10. Taranta A. Fortunati D., Longo M., Rucci N., lacomino E., Aliberti F., et al. Imbalance of osteoclastogenesis-regulating factors in patients with coeliac disease. J Bone Miner Res 2004; 19:1112-21.
(32.2\%). The association of clinical, biological and radiologic rickets signs in 30\% of patients highlight the role of phosphor-calcium metabolism alteration impact in the pathogeny of osteopenia.

$25(\mathrm{OH})$ vitamin $\mathrm{D}$ values follow up, vitamin $\mathrm{D}$ deficiency marker, is useful for the conduction of treatment in patients with malabsorption syndromes. Its value didn't correlate with bone density evaluation by QUS in this study.

The negative correlation of ultrasonographic bone parameters with inflammation markers and anti-transglutaminase antibodies values require that Osteodensitometry is performed and the inclusion of osteopenia as a witness of the inflammatory immunological process.

11. Sugai E., Chernavsky A., Pedreira S., Smecuol E., Vazquez H., Niveloni S., Mazure R., Mauriro E., Rabinovich G.A., J.C. Bai. Bone-specific antibodies in sera from patients with celiac disease: characterization and implications in osteoporosis. J Clin Immunol 2002; 22(6): 353-62.

12. Fok J., Brown N.E., Zuberbuhler P., Tabak J., Tom M. Low bone mineral density in cystic fibrosis patients. Can J Diet Pract Res 2002:63(4):192-197.

13. Sood M., Hambleton G., Super M., Fraser W.D., Adams J.E., Mughal M.Z. Bone status in cystic fibrosis. Arch Dis Child 2001:84:516-520.

14. Putman M.S., Baker J.F., Uluer A., et al. Trends in Bone Mineral Density in Young Adults with Cystic Fibrosis over a 15 Year Period. J Cyst Fibros 2015: 14(4): 526-532.

15. Salamoni F., Roulet M., Gudinchet F., Pilet M., Thiébaud D., Burckhardt P. Bone mineral content in cystic fibrosis patients: correlation with fat free mass. Arch Dis Child 1997:74(4):314-318

16. Bhudhikanok G.S., Wang M.C., Marcus R., Harkins A., Moss R.B., Bachrach L.K. Bone acquisition and loss in children and adults with cystic fibrosis: a longitudinal study. J Pediatr 1998:133(1):18-27.

17. Conway S.P., Morton A.M., Oldroyd B., Truscott J.G., White H., Smith A.H., et al. Osteoporosis and osteopenia in adults and adolescents with cystic fibrosis: prevalence and associated factors. Thorax. 2000: 55(9):798-804. 